\title{
Perfil de humor e fatores associados em participantes de um programa de reabilitação cardiopulmonar e metabólica
}

\author{
Profile of mood and associated factors among participants of a \\ program of cardiopulmonary and metabolic rehabilitation
}

Ricardo Brandt', Maick da Silveira Viana', Ricardo de Azevedo Klumb Steffens', Rodrigo Batalha Silva', Sabrina Weiss Sties², Ana Inês Gonzáles², Tales de Carvalho², Alexandro Andrade?

\section{RESUMO}

Objetivo: Analisar o perfil de humor e fatores associados em participantes de um programa de reabilitação cardiopulmonar e metabólica. Método: Cento e quarenta e quatro pacientes responderam a um questionário de caracterização e à Escala de Humor de Brunel (BRUMS). O questionário de caracterização foi constituído de perguntas sobre dados pessoais e clínicos, enquanto o BRUMS avaliou o humor por meio de seis fatores: tensão, depressão, raiva, vigor, fadiga e confusão. Os dados foram tratados com estatística descritiva e inferencial (a =0,05). Resultados: De maneira geral, os pacientes com doenças cardiovasculares e metabólicas apresentaram boa saúde mental, com níveis elevados de vigor, associados à moderada tensão, depressão, raiva, fadiga e confusão. Os participantes idosos apresentaram menor tensão, raiva, fadiga e confusão do que os adultos, enquanto os participantes com dislipidemia tinham maior tensão. Aqueles com insuficiência cardíaca eram mais tensos e fadigados. Quem fuma apresentou menor vigor e maior tensão, depressão e raiva. Conclusão: Pacien-

\section{Palavras-chave}

Cardiopatia, saúde mental, humor. tes com doenças cardiovasculares participantes de um programa de reabilitação apresentaram boa saúde mental na avaliação realizada, e os participantes adultos, com dislipidemia, insuficiência cardíaca e que fumam tinham humor mais deprimido.

\section{ABSTRACT}

Objective: To examine the profile of mood states and associated factors in participants of program of cardiopulmonary and metabolic rehabilitation. Method: One hundred and fortyfour patients answered a characterization questionnaire and the Brunel Mood Scale (BRUMS). The characterization questionnaire consisted of questions about personal and clinical data, and the BRUMS evaluates the mood by six factors: tension, depression, anger, vigor, fatigue and confusion. The data were processed by descriptive statistics and by the Mann-Whitney test and inferential $(a=0,05)$. Results: In general, patients with heart and metabolic diseases had good mental health, with high levels of vigor associated with low levels of tension, depression, anger, fatigue and confusion. Older participants showed higher levels of tension, anger, fatigue and confusion, while participants who have heart failure showed worse

1 Universidade do Estado de Santa Catarina (Udesc), Centro de Ciências da Saúde e do Esporte (Cefid), Laboratório de Psicologia do Esporte e do Exercício (Lape)

2 Universidade do Estado de Santa Catarina (Udesc), Centro de Ciências da Saúde e do Esporte (Cefid), Núcleo de Cardiologia e Medicina do Exercício (NCME).

Endereço para correspondência: Ricardo Brandt Centro de Ciências da Saúde e do Esporte, Laboratório de Psicologia do Esporte e do Exercício Rua Pascoal Simone, 358, Coqueiros

88080-350 - Florianópolis, SC

E-mail: ricabrandt@gmail.com 


\section{Keywords}

Cardiopathy, mental

health, mood. levels of tension and fatigue. Smokers had lower vigor and increased tension, depression and anger. Conclusion: Patients with heart disease participating in a rehabilitation program showed good mental health evaluation. Adults participants, with dyslipidemia, heart failure and smokers have more depressed mood.

\section{INTRODUÇÃO}

Nas últimas décadas, as doenças crônicas não transmissíveis, em especial as cardiovasculares, têm recebido atenção especial dos profissionais da saúde por causa das elevadas taxas de morbimortalidade. Tais doenças são as principais causas de morte no mundo, afetando igualmente homens e muIheres; mais de $80 \%$ desses óbitos ocorrem em países com rendimento econômico baixo e médio'.

As doenças cardiovasculares caracterizam-se por mudanças na homeostase, função neuroendócrina e, eventualmente, por limitações físicas, o que pode acarretar desordens do humor $^{2}$. Além disso, fatores de risco como sexo, idade, obesidade, dislipidemia, diabetes, hipertensão arterial, tabagismo e sedentarismo estão associados também aos estados de humor ${ }^{3-9}$.

As desordens psiquiátricas nesse grupo de pacientes vêm sendo estudadas há muito tempo ${ }^{6}$, pois possuem importante prevalência e levam ao aumento do consumo de medicamentos ${ }^{10}$. As desordens do humor têm importante influência na evolução das doenças cardiovasculares. Por exemplo, pacientes que sofrem de depressão após um evento cardíaco apresentam maiores taxas de morbidade e mortalidade quando comparados aos não deprimidos ${ }^{11,12}$.

O humor reflete um padrão complexo de comportamentos, sentimentos, pensamentos, estados corporais e emocionais $^{13}$. Os estados de humor incluem uma série de fatores psicológicos, como a depressão do humor, a raiva e a confusão mental, e fatores psicossomáticos, como a fadiga, o vigor e a tensão ${ }^{14,15}$. Segundo Morgan ${ }^{16}$, baixos níveis de tensão, depressão, raiva, fadiga e confusão mental associados a um vigor elevado representam um estado de saúde mental positivo.

Nesse contexto, esta pesquisa teve por objetivo analisar o perfil de humor e fatores associados em participantes de um programa de reabilitação cardiopulmonar e metabólica. Tal programa destaca a prática de exercícios físicos, que, em decorrência de sua ação ansiolítica e antidepressiva, pode neutralizar os efeitos adversos das doenças cardiovasculares e metabólicas sobre o humor.

\section{MÉTODO}

O projeto de pesquisa foi aprovado pelo Comitê de Ética em Pesquisa em Seres Humanos da Universidade do Estado de Santa Catarina (protocolo: 149/2011). Todos os participantes assinaram o Termo de Consentimento Livre e Esclarecido
(TCLE), o qual explicitava todos os procedimentos da pesquisa e o caráter voluntário dela.

\section{Participantes}

Participaram da pesquisa 144 pacientes, sendo 77 homens e 67 mulheres, com idade entre 39 e 87 anos ( $\bar{x}=64,6 \pm 10,1$ ). Todos são participantes regulares, há pelo menos seis meses, de um programa de extensão na universidade que promoveu o estudo. O programa oferece tratamento direcionado a pacientes com doenças cardiovasculares e/ou metabólicas por meio da prática de exercícios físicos.

\section{Instrumentos}

Foram utilizados como instrumentos: questionário de caracterização dos participantes e Escala de Humor de Brunel.

O questionário de caracterização dos participantes foi constituído por perguntas que abordaram aspectos relacionados às características sociodemográficas (sexo e idade) e fatores de risco cardiovascular (dislipidemia, diabetes, pressão alta, doença arterial obstrutiva periférica, hipertensão arterial sistêmica e tabagismo).

A Escala de Humor de Brunel (BRUMS) é uma versão reduzida do Profile of Mood States (POMS), desenvolvida por Terry et al. ${ }^{17}$, e avalia seis estados de humor (tensão, depressão, raiva, vigor, fadiga e confusão mental - tabela 1) em populações de adultos e adolescentes. O questionário é composto por 24 escalas de cinco níveis ( 0 = nada e 4 = extremamente), as quais devem ser respondidas pelo participante considerando como ele se sente no momento da avaliação. Com a soma das respostas referentes a cada construto, obtém-se um escore que varia de 0 a 16 para cada estado de humor (por exemplo: esgotado + exausto + sonolento + cansado = fadiga). O BRUMS foi validado para o Brasil por Rohlfs ${ }^{18}$, com valores de consistência interna (alfa de Cronbach) superiores a 0,70 para todos os construtos da escala.

Tabela 1. Subescalas da Escala de Humor de Brunel

\begin{tabular}{ll}
\hline Subescalas & Definição \\
\hline Tensão & Estado de tensão musculoesquelético e preocupação \\
Depressão & Estado emocional de desânimo, tristeza, infelicidade \\
Raiva & Estado de hostilidade, relativamente aos outros \\
Vigor & Estado de energia, vigor físico \\
Fadiga & Estado de cansaço, baixa energia \\
Confusão mental & Estado de atordoamento, instabilidade nas emoções
\end{tabular}

Fonte: Brandt R, Viana MS, Segato L, Andrade A. Estados de humor de velejadores durante o Pré-Panamericano. Motriz. 2010;16(4):834-40 


\section{Procedimentos}

As coletas de dados aconteceram durante três dias, mediante agendamento prévio de cada participante. Os instrumentos foram aplicados em um ginásio de esportes da universidade que promoveu o estudo, no qual foi organizada uma bateria de avaliações com a participação de 12 pesquisadores devidamente treinados para aplicação dos respectivos testes de sua responsabilidade. Com grande espaçamento entre as estações, garantiram-se coletas sem interferências de terceiros e conforto aos participantes.

\section{Tratamento dos dados}

Em relação à estatística descritiva, os dados foram analisados a partir da distribuição de frequências, médias, percentuais e desvios-padrão. Quanto à estatística inferencial, por se tratarem de dados com distribuição não paramétrica (Kolmogorov-Smirnov < 0,05), foram realizados os testes de Mann-Whitney e Kruskal-Wallis, visando comparar os estados de humor de grupos com diferentes características. Estabeleceu-se valor a de 0,05 como nível de significância $(p<0,05)$.

\section{RESULTADOS}

Em relação aos fatores de risco cardiovascular, $16,4 \%$ dos participantes tinham dislipidemia, 20,7\%, diabetes e 59,7\%, hipertensão arterial sistêmica. A doença arterial obstrutiva periférica esteve presente em 9,3\% dos casos, a insuficiência cardíaca em $15,7 \%$ e a doença arterial coronariana em $54,3 \%$. Quanto ao tabagismo, 54,0\% nunca haviam fumado, 39,6\% eram ex-fumantes e 6,4\% mantinham o hábito de fumar.

De maneira geral, os pacientes apresentaram elevado vigor e moderada tensão, depressão, raiva, fadiga e confusão. Embora tenham apresentado algumas diferenças nas médias, homens e mulheres não se diferenciaram significativamente em relação aos estados de humor. Ao se compararem os estados de humor de adultos (com menos de 60 anos) e idosos (com 60 anos ou mais), foram verificadas diferenças significativas entre os grupos. Adultos apresentaram níveis mais elevados de tensão, raiva, fadiga e confusão do que os idosos (Tabela 2).

Tabela 2. Estados de humor geral e sua comparação entre grupos de homens e mulheres, adultos e idosos, e com a presença ou não dos seguintes fatores de risco: dislipidemia, diabetes, doença arterial obstrutiva periférica, insuficiência cardíaca, doença arterial coronariana, hipertensão arterial sistêmica e tabagismo $(\bar{x} / \pm)^{\dagger}$

\begin{tabular}{|c|c|c|c|c|c|c|c|c|c|c|c|c|}
\hline & \multicolumn{2}{|c|}{ Tensão } & \multicolumn{2}{|c|}{ Depressão } & \multicolumn{2}{|c|}{ Raiva } & \multicolumn{2}{|c|}{ Vigor } & \multicolumn{2}{|c|}{ Fadiga } & \multicolumn{2}{|c|}{ Confusão } \\
\hline & $\bar{x}$ & \pm & $\bar{x}$ & \pm & $\bar{x}$ & \pm & $\bar{x}$ & \pm & $\bar{x}$ & \pm & $\bar{x}$ & \pm \\
\hline Média geral dos fatores de humor $(n=144)$ & 3,6 & 3,7 & 2,0 & 3,2 & 2,0 & 3,5 & 9,8 & 2,8 & 3,6 & 2,8 & 2,1 & 3,0 \\
\hline \multicolumn{13}{|l|}{ Sexo } \\
\hline Homens $(n=77)$ & 3,5 & 3,4 & 1,7 & 2,8 & 2,3 & 3,7 & 9,9 & 3,1 & 3,1 & 3,2 & 1,9 & 2,4 \\
\hline Mulheres $(n=67)$ & 3,7 & 4,1 & 2,4 & 3,7 & 1,7 & 3,3 & 9,7 & 2,6 & 4,1 & 4,4 & 2,3 & 3,4 \\
\hline Grupo de idade & $*$ & & & & * & & & & * & & * & \\
\hline Até 60 anos $(n=45)$ & 4,7 & 4,1 & 2,7 & 3,9 & 2,8 & 3,8 & 9,8 & 3,0 & 4,4 & 3,7 & 3,1 & 3,4 \\
\hline Com mais de 60 anos $(n=95)$ & 2,9 & 3,3 & 1,6 & 2,8 & 1,5 & 3,0 & 9,7 & 2,7 & 3,2 & 3,9 & 1,7 & 2,5 \\
\hline Dislipidemia & * & & & & & & & & & & & \\
\hline $\operatorname{Sim}(n=23)$ & 3,9 & 4,1 & 2,3 & 3,3 & 2,6 & 4,0 & 9,5 & 2,8 & 4,0 & 4,1 & 2,6 & 3,2 \\
\hline Não $(n=117)$ & 3,2 & 3,3 & 1,7 & 3,1 & 1,4 & 2,8 & 10 & 2,8 & 3,2 & 3,2 & 1,8 & 2,4 \\
\hline \multicolumn{13}{|l|}{ Diabetes } \\
\hline $\operatorname{Sim}(n=43)$ & 3,2 & 3,4 & 1,5 & 2,4 & 1,6 & 2,5 & 10 & 2,9 & 3,3 & 4,1 & 2,0 & 2,6 \\
\hline Não $(n=97)$ & 3,7 & 3,8 & 2,2 & 3,5 & 2,2 & 3,8 & 9,5 & 2,8 & 3,7 & 3,7 & 2,2 & 3,0 \\
\hline \multicolumn{13}{|l|}{ Hipertensão arterial sistêmica } \\
\hline $\operatorname{Sim}(n=83)$ & 3,5 & 3,8 & 2,1 & 3,4 & 2,0 & 3,6 & 9,4 & 2,6 & 3,6 & 4,1 & 2,1 & 2,8 \\
\hline Não $(\mathrm{n}=56)$ & 3,8 & 3,7 & 1,8 & 2,8 & 2,0 & 3,4 & 9,9 & 2,8 & 3,6 & 3,4 & 2,2 & 2,9 \\
\hline \multicolumn{13}{|l|}{ Doença arterial obstrutiva periférica } \\
\hline $\operatorname{Sim}(n=13)$ & 3,6 & 3,8 & 2,8 & 3,8 & 1,6 & 2,6 & 9,2 & 3,4 & 4,1 & 3,5 & 3,6 & 4,4 \\
\hline Não $(n=127)$ & 3,6 & 3,8 & 1,9 & 3,1 & 2,0 & 3,6 & 9,7 & 2,7 & 3,5 & 3,8 & 2,0 & 2,6 \\
\hline Insuficiência cardíaca & $*$ & & & & & & & & * & & & \\
\hline $\operatorname{Sim}(n=22)$ & 5,6 & 4,3 & 2,5 & 3,5 & 3,4 & 4,7 & 10 & 3,3 & 5,3 & 4,1 & 2,8 & 2,9 \\
\hline Não $(n=118)$ & 3,2 & 3,5 & 1,9 & 3,1 & 1,7 & 3,2 & 9,6 & 2,6 & 3,3 & 3,7 & 2,0 & 2,8 \\
\hline \multicolumn{13}{|l|}{ Doença arterial coronariana } \\
\hline $\operatorname{Sim}(n=76)$ & 3,4 & 3,3 & 1,7 & 2,8 & 1,8 & 3,1 & 3,4 & 3,3 & 3,1 & 3,6 & 2,0 & 2,5 \\
\hline Não $(\mathrm{n}=64)$ & 3,8 & 4,2 & 2,3 & 3,1 & 2,2 & 3,9 & 9,9 & 2,6 & 4,2 & 4,1 & 2,3 & 3,1 \\
\hline Tabagismo & $*$ & & * & & $*$ & & * & & & & & \\
\hline Nunca fumou $(n=75)$ & 3,0 & 3,7 & 1,8 & 3,3 & 1,5 & 3,2 & 9,9 & 2,7 & 3,1 & 3,9 & 1,7 & 2,8 \\
\hline Ex-fumante $(n=55)$ & 3,6 & 3,4 & 1,8 & 2,6 & 2,5 & 3,7 & 10,0 & 2,7 & 4,1 & 3,6 & 2,3 & 2,8 \\
\hline Fumante $(\mathrm{n}=9)$ & 7,1 & 3,6 & 3,7 & 3,4 & 4,1 & 4,8 & 7,3 & 2,8 & 4,2 & 2,3 & 3,3 & 2,9 \\
\hline
\end{tabular}

* Diferença significativa ao nível de $p<0,05$.

† Pela existência de missings, existem casos em que a soma das categorias não resulta no " $n$ " total. 
Foram comparados pacientes que possuíam ou não os riscos cardiovasculares investigados. Pacientes com dislipidemia apresentaram maior tensão do que seus pares sem essa condição. Verificaram-se diferenças significativas também entre aqueles que tinham ou não insuficiência cardíaca, e os acometidos por esta tiveram maior tensão e fadiga. Quanto ao tabagismo, os fumantes apresentaram níveis significativamente maiores de tensão, depressão e raiva e menor nível de vigor do que aqueles que pararam de fumar ou que nunca fumaram (Tabela 2).

Os demais grupos comparados não se diferenciaram em relação aos estados de humor, embora sejam percebidas algumas diferenças na análise descritiva (Tabela 2).

\section{DISCUSSÃO}

Este estudo teve por objetivo analisar o perfil de humor e fatores associados em participantes de um programa de reabilitação cardiopulmonar e metabólica. Além das implicações físicas, para as quais geralmente são direcionadas mais atenção, torna-se importante considerar que pessoas nessa condição de saúde também podem ter a saúde mental prejudicada.

Os estados de humor são sentimentos subjetivos, tendo duração variável, de algumas horas a alguns dias, podendo refletir sentimentos de exaltação ou felicidade, tristeza, angústia, entre outros ${ }^{15}$. Os pacientes apresentaram vigor elevado, associado a níveis moderados de tensão, depressão, raiva, fadiga e confusão, o que não indica um estado de humor deprimido. De maneira geral, esses resultados corroboram o modelo de saúde mental proposto por Morgan ${ }^{16}$, sugerindo que os pacientes que participaram da pesquisa se encontram em boas condições de saúde mental.

De acordo com Frasure-Smith et al. ${ }^{19}$, alterações de humor podem ter um impacto negativo na saúde física, como a diminuição da taxa de sobrevivência após infarto do miocárdio para pessoas com depressão. Nesse sentido, é importante que haja atenção especial para as alterações de humor de pacientes cardíacos, inclusive com acompanhamento psicológico ou psiquiátrico ${ }^{20}$.

Os transtornos psiquiátricos têm elevada prevalência em pacientes cardiopatas que frequentam programas de reabilitação ${ }^{10}$, bem como naqueles com síndrome metabólica ${ }^{5}$. Segundo Sardinha et al.10, a prática de exercícios físicos pode ter efeito regulador do humor, o qual pode ser explicado pelos efeitos da prática regular de exercícios físicos sobre o sistema serotoninérgico9 ${ }^{9}$. Tendo em vista que os participantes da pesquisa são pacientes de um programa de reabilitação embasado especialmente na prática de exercícios físicos, é possível que os bons resultados apresentados nos estados de humor sejam em parte advindos dessa prática, embora não seja possível confirmar essa hipótese por causa da caracterís- tica transversal da pesquisa. Pesquisas futuras, com característica experimental, podem melhor elucidar a influência da prática de exercícios nos estados de humor dessa população.

No presente estudo, pacientes idosos apresentaram tensão, raiva, fadiga e confusão significativamente inferiores aos adultos. A tensão refere-se à alta tensão musculoesquelética, a qual pode ser observada por meio de manifestações psicomotoras como agitação e inquietação ${ }^{21}$. A raiva descreve sentimentos de hostilidade relacionados à antipatia aos outros e a si mesmo, com uma escala de sentimentos que variam de leve irritação até a cólera, associados a estímulos do sistema nervoso autônomo ${ }^{22}$. A fadiga representa um estado de esgotamento, apatia e baixo nível de energia ${ }^{14}$. A confusão pode ser caracterizada como um atordoamento, uma resposta à ansiedade e à depressão. Sentimentos de incerteza, instabilidade para o controle de emoções e atenção também caracterizam a confusão mental ${ }^{23}$. Duas hipóteses podem justificar esses resultados, tendo em vista as características das variáveis descritas. Primeiramente, é possível que uma pessoa mais jovem seja mais impactada pela descoberta e convívio com uma doença cardiovascular ou metabólica, pois normalmente essas doenças acometem pessoas em faixa etária mais elevada. Além disso, convivendo por mais tempo com a doença, idosos podem saber lidar emocionalmente de maneira mais adequada com as situações decorrentes dela, diminuindo a influência delas sobre o humor. Dessa forma, atenção psicológica especial deve ser dada para pessoas mais jovens que venham a sofrer com alguma doença cardíaca ou metabólica, pois se trata de um grupo, ao que parece, mais suscetível a alterações no humor em decorrência dessa condição de saúde.

Os pacientes com insuficiência cardíaca apresentam pior estado de humor, com níveis significativamente superiores de tensão e fadiga. A mesma tendência foi observada para os demais fatores, porém sem significância estatística. A insuficiência cardíaca é caracterizada por dispneia e fadiga, o que pode promover diminuição do estado de saúde física e social, bem como prejuízos em outras dimensões relacionadas à qualidade de vida ${ }^{24}$. Pacientes com IC sofrem mais com distúrbios de humor do que indivíduos saudáveis, no entanto, de modo geral, o estado psicológico destes é semelhante ao de pacientes com outras doenças crônicas graves ${ }^{25}$. Os fatores em que participantes com IC se diferenciaram, tensão e fadiga, são considerados psicossomáticos ${ }^{14,15}$, sobre os quais a prática de exercícios físicos poderia trazer benefícios de forma mais direta. Porém, mesmo participando de um programa que promove a prática de exercícios físicos, pacientes com IC demonstraram humor mais deprimido em comparação aos demais. Visando maiores benefícios também para pacientes com IC, sugere-se que diferentes formas de treinamento (com diferentes intensidades, por exemplo) sejam investigadas para melhor atender esse grupo, não desconsiderando que a fadiga é inerente à IC. 
O presente estudo demonstrou que as pessoas que nunca fumaram, ou eram ex-fumantes, apresentaram menor tensão, depressão e raiva, e maior vigor do que os fumantes. Esses resultados divergem dos achados de Elliot et al. ${ }^{11}$, que, estudando pacientes cardíacos que seriam submetidos à cirurgia, verificaram que os ex-fumantes eram mais confusos, fadigados e tinham menor vigor. Percebe-se que o uso de tabaco é um fator negativo aos estados de humor, prejudicando a saúde mental dos pacientes com doenças cardiovasculares e metabólicas. Conforme Glassman et al. ${ }^{\circ}$, essas alterações do humor podem estar associadas com dificuldades em parar de fumar ou adotar um estilo de vida mais saudável. Dessa forma, sugere-se que uma das prioridades para pessoas com doenças cardiovasculares ou metabólicas seja não fumar. Essa orientação do profissional que estiver envolvido com o atendimento ao paciente é útil não somente contra os malefícios do tabagismo à saúde física, e inclusive cardiovascular, já suficientemente descritos na literatura, mas também visando a benefícios para a sua saúde mental. Por outro lado, parar de fumar também é um fator estressante ao tabagista, necessitando de acompanhamento psicológico com o objetivo de uma mudança comportamental que seja saudável, nesse que é um momento bastante delicado.

Sugere-se que pesquisas futuras lancem mão de alguma medida biológica, como o cortisol, por exemplo, pois estudos apontam que essas medidas são mais válidas do que instrumentos que utilizam medidas retrospectivas de humor, como o POMS, o PANAS 26,27 e o BRUMS. Por outro lado, a realização de avaliações longitudinais poderia minimizar esse viés, bem como conhecer os aspectos socioafetivos dos pacientes, com a intenção de diminuir as chances de erro na interpretação dos dados. Esse cuidado deve ser dado especialmente nas variáveis negativas do humor, como raiva, fadiga e confusão, pois podem sofrer alterações em decorrência de situações do cotidiano ${ }^{11}$.

\section{CONCLUSÃO}

Os participantes com doenças cardiovasculares e metabólicas apresentaram boa saúde mental no momento da avaliação realizada, pois seus estados de humor foram compostos por um elevado vigor associado a níveis toleráveis de tensão, depressão, raiva, fadiga e confusão. Em relação às variáveis associadas aos estados de humor, verificou-se que os participantes idosos apresentaram menor tensão, raiva, fadiga e confusão do que os adultos. Aqueles que sofriam com a insuficiência cardíaca tiveram maior tensão e fadiga, e os com dislipidemia, maior tensão. Quanto ao tabagismo, os que fumavam tiveram maior tensão, depressão e raiva, além de menor vigor do que os que não fumavam ou eram ex-fumantes.
A participação em programas de exercícios físicos vem sendo considerada importante para pacientes cardiopatas, especialmente pelos benefícios cardiopulmonares. Sugere-se que os estados de humor positivos verificados nos participantes da pesquisa, de maneira geral, podem estar relacionados ao programa de exercícios físicos que estão envolvidos, sendo essas práticas um possível meio de promoção também da saúde mental. Cabe destacar que alguns grupos específicos não apresentaram dados tão positivos (adultos, com IC, fumantes), cabendo estudos mais específicos sobre eles.

A presente pesquisa propõe também o BRUMS como um instrumento útil para a avaliação de pessoas com doenças cardiovasculares e metabólicas, abordando a percepção dos avaliados sobre sua raiva, tensão, depressão, vigor, fadiga e confusão e tornando-se um instrumento útil para futuras pesquisas que buscam investigar a saúde mental em populações com características semelhantes.

\section{REFERÊNCIAS}

1. World Health Organization. The World Health Report 2005: health systems: improving performance. Geneva: WHO; 2005.

2. Grippo AJ, Johnson AK. Stress, depression, and cardiovascular dysregulation: a review of neurobiological mechanisms and the integration of research from preclinical disease models. Stress. 2009;12(1):1-21.

3. Duits AA, Duivenvoorden HJ, Boeke S, Taams MA, Mochtar B, Krauss XH, et al. The course of anxiety and depression in patients undergoing coronary artery bypass graft surgery. J Psychosom Res. 1998:45(2):127-38.

4. Davidson RJ, Lewis DA, Alloy LB, Amaral DG, Bush G, Cohen JD, et al. Neural and behavioral substrates of mood and mood regulation. Biol Psychiatry. 2002;52(6):478-502.

5. Teixeira PJR, Rocha FL. Associação entre síndrome metabólica e transtornos mentais. Rev Psiq Clín. 2007;34(1):28-38.

6. Bankier B, Januzzi IL, Littman AB. The high prevalence of multiple psychiatric disorders in stable outpatients with coronary heart disease. Psychosom Med. 2004;66(5):645-50.

7. Frasure-Smith N, Lesperance F, Juneau M, Talajic M, Bourassa MG. Gender, depression, and one-year prognosis after myocardial infarction. Psychosom Med. 1999;61:26-37.

8. Glassman AH, Covey LS, Stetner F, Rivelli S. Smoking cessation and the course of major depression: a follow-up study. Lancet. 2001;357:1929-32.

9. Peluso MAM, Andrade LHG. Physical activity and mental health: the association between exercise and mood. Clinics. 2005;60(1):61-70.

10. Sardinha A, Araújo CGS, Silva ACO, Nardin AE. Prevalência de transtornos psiquiátricos e ansiedade relacionada à saúde em coronariopatas participantes de um programa de exercício supervisionado. Rev Psiq Clín. 2011;38(2):61-5.

11. Elliot PC, Murphy BM, Oster KA, Le Grande MR, Higgins RO, Worceste MUC. Changes in mood states after coronary artery bypass graft surgery. Eur J Cardovasc Nursing. 2010;:188-94.

12. Blumenthal JA, Lett HS, Babyak MA, White W, Smith PK, Mark DB, et al. Depression as a risk factor for mortality after coronary artery bypass surgery. Lancet. 2003;362:604-9.

13. Wielenska RC. Terapia comportamental do transtorno obsessivo-compulsivo. Rev Bras Psiquiatr. 2001;23(II):62-4.

14. Brandt $R$, Fonseca ABP, Oliveira LGA, Steffens RAK, Viana MS, Andrade A. Perfil de humor de mulheres com fibromialgia. J Bras Psiquiatr. 2012;60(3):216-20.

15. Brandt $R$, Viana MS, Segato L, Andrade A. Estados de humor de velejadores durante o Pré-Panamericano. Motriz. 2010;16(4):834-40.

16. Morgan WP. Test of champions: the iceberg profile. Psychol Today. 1980;39:92-108. 
17. Terry PC, Lane AM, Fogarty GJ. Construct validity of the Profile of Mood States - adolescents for use with adults. Psychol Sport Exerc. 2003;4(2):125-39.

18. Rohlfs ICPM. Validação do teste de BRUMS para avaliação de humor em atletas e não atletas brasileiros. 2006. Dissertação (Mestrado em Ciências do Movimento Humano) - Universidade do Estado de Santa Catarina. Florianópolis (SC): UDESC; 2006.

19. Frasure-Smith N, Lesperance F, Talajic M. Depression following myocardial infarction: impact on 6-month survival. JAMA. 1993;270:1819-25.

20. While A, Keen L. The effects of statins on mood: a review of the literature. Eur J Cardiovasc Nurs. 2010;1:1-12.

21. Terry PC. The efficacy of mood state profiling among elite performers: a review and synthesis. Sport Psychol. 1995;9(3):309-24.

22. Schinka JA, Velicer WF, Weiner IB. Handbook of psychology: research methods in psychology. New Jersey: Wiley; 2003.
23. Beck AT, Clark DA. Anxiety and depression: an information processing perspective. Anxiety Res. 1998;1(1):23-56.

24. Krumholz HM, Butler J, Miller J, Vaccarino V, Williams CS, Leon CFM, et al. Prognostic importance of emotional support for elderly patients hospitalized with heart failure. Circulation. 1998:97(10):958-64.

25. Majani G, Callegari S, Pierobon A, Viola L, Manera M, Opasich C, et al. The psychological side of chronic heart failure. A pluriannual experience. G Ital Cardiol. 1997;27:244-54.

26. Bostock S, Hamer M, Andrew J, Wawrzyniak J, Mitchell ES, Steptoe A. Positive emotional style and subjective, cardiovascular and cortisol responses to acute laboratory stress. Psychoneuroendocrinology. 2011;36:1175-85.

27. Steptoe A, Gibson L, Hamer M, Wardle J. Neuroendocrine and cardiovascular correlates of positive affect measured by ecological momentary assessment and by questionnaire. Psychoneuroendocrinology. 2007;32:56-64. 